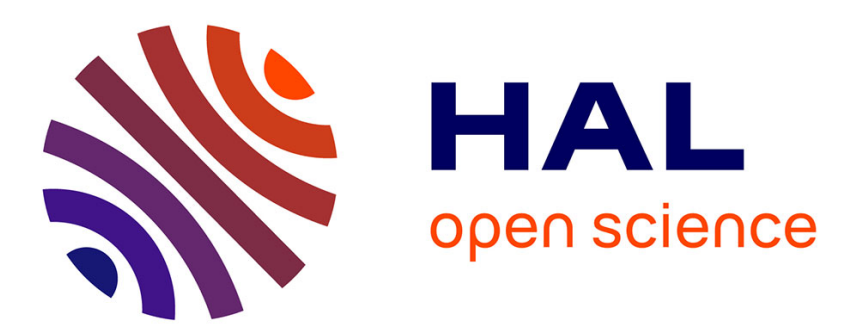

\title{
Optimization of direction finders by Genetic Algorithms
}

Lionel Taïeb, Marc Schoenauer

\section{To cite this version:}

Lionel Taïeb, Marc Schoenauer. Optimization of direction finders by Genetic Algorithms. Proc. GALESIA, First IEE/IEEE International Conference on Genetic ALgorithms in Engineering Systems: Innovations and Applications, Sep 1995, Sheffield, United Kingdom. hal-02986280

\section{HAL Id: hal-02986280 \\ https://hal.inria.fr/hal-02986280}

Submitted on 2 Nov 2020

HAL is a multi-disciplinary open access archive for the deposit and dissemination of scientific research documents, whether they are published or not. The documents may come from teaching and research institutions in France or abroad, or from public or private research centers.
L'archive ouverte pluridisciplinaire HAL, est destinée au dépôt et à la diffusion de documents scientifiques de niveau recherche, publiés ou non, émanant des établissements d'enseignement et de recherche français ou étrangers, des laboratoires publics ou privés. 


\section{Optimization of direction finders by Genetic Algorithms}

\author{
L. Taïeb ${ }^{1,2}$ \\ 1 THOMSON-CSF (RCM division) \\ La Clef Saint-Pierre \\ 1 Boulevard Jean Moulin \\ 78852 Elancourt Cedex, France
}

\author{
M. Schoenauer ${ }^{2}$ \\ ${ }^{2}$ Centre de Mathématiques Appliquées, \\ Ecole Polytechnique, \\ 91128 Palaiseau Cedex, France \\ FirstName.Name@polytechnique.fr
}

\begin{abstract}
1 The relative positions of the antennas of a direction finder are critical regarding the efficiency and the robustness of the indications of the apparatus. The state of the art in direction finder optimization is limited with classical methods to the case of direction finders with 3 antennas, with naive trialand-error methods for more complex cases. The use of Genetic Algorithms brings a tremendous increase to that domain, allowing the optimization of direction finders with up to 10 antennas in a reasonable computing time. Moreover, as GAs provide multiple optimal solutions on multi-modal problems, a thorough numerical investigation of all possible optima provides a new insight in the underlying optimization problem, finally leading to the derivation of a formula for the optimal direction finders.
\end{abstract}

\section{Introduction}

A direction finder is a measuring apparatus used to determine the bearing angle (or site angle) of the direction of an electromagnetic source from the differences in phase measured between couples of antennas. It is used by airplanes or ships for the determination of the position of radars or radio beacons. Direction finders used for radar detection and localization, with high frequency signals, are studied and built by the division "Radars et Contre-Mesures" (RCM) of the French firm THOMSON.

Even in the simplest case of aligned antennas, in which a single direction is sought, the exact positions of the antennas is a critical factor of the efficiency and accuracy of the direction finder ([10]). This efficiency is quantified by a maximum allowable error, the amount of noise that a given direction finder can afford while still giving a correct answer regarding the direction of the incoming signal. The case of 3 antennas has been well studied ([15]), and analytical formulas can be derived for such an

\footnotetext{
${ }^{1}$ Submitted to GALESIA'95, First IEE/IEEE International Conference on Genetic ALgorithms in Engineering Systems: Innovations and Applications.
}

error, allowing brute optimization: both extremal antennas are fixed to the prescribed total length of the apparatus, and every possible placement of the third antenna on a fixed grid between the two others can be investigated.

But such a method does not scale up: even in the case of four antennas, the classical method is to start from the optimal solution of the corresponding 3antennas problem, and to add the fourth one by trials and errors, eventually moving step by step the third antenna. And of course the global optimization of more than 4 antennas is unconceivable with such methods.

The reasons for that are both: First, the function to optimize is not analytically known, and is accessible only through numerical computation. Second, it presents a very large number of local optima, and the standard deterministic optimization methods are known to generally fall into one of these local optima, unless some good starting points can be guessed. The use of stochastic optimization methods in such situations seems quite natural.

First introduced by Holland [4], then popularized by Goldberg [2], Genetic Algorithms (GAs) are evolutionary algorithms crudely mimicking natural evolution. They are based on the Darwinian principle of the Survival of the fittest: a population of chromosomes (points of the search space) undergoes a succession of generations, and each chromosome is applied successively the genetic operators of selection, cross-over and mutation. GAs, though they are not function optimizers ([5]) have demonstrated they can be used as trustworthy optimization methods, particularly in cases where other classical methods fail. The price to pay for that is a fairly high computational cost.

This paper presents results obtained by GAs on the problem of optimizing a direction finder, problem which is introduced in Section 2. The main difficulties come from the noise in the measures made by the antennas, which cannot be avoided in real world applications. The mathematical optimization problem, taking these errors into account, is posed, but all the technical details are omitted. Three annexes at the end of the paper give the most important calculation details, and all detailed 
demonstrations can be found in [15]. Section 3 gives a brief overview of the genetic algorithm used on the direction finder problem, while section 4 presents the results obtained in the case of aligned antennas. Direction finders up to 10 antennas can be optimized by this method.

Moreover, multiple optimal solutions can be found by GAs, either in multiple runs, or using niching schemes like the well-known sharing scheme ([3]). And a careful look at all solutions found by the GAs in the case of 3 antennas for different values of the total length $D$ of the direction finder surprisingly lead to a relation linking the optima for lengths $D$ and $D / 2$, thus completely solving the 3 antennas case. Section 5 presents this unusual approach, where numerical computation through evolutionary computation happened to be the necessary step toward the theoretical comprehension of the underlying phenomenon. The final section presents some preliminary results obtained in the more general case of coplanar antennas (not aligned). The extension of the method to that case is conceptually straightforward, though many technical difficulties, not presented here, arise during the derivation of the fitness function. Finally, some future directions of research are sketched, in the area of electromagnetic engineering using GA optimization.

\section{Background}

A direction finder is made of several antennas, recording electromagnetic signals coming from a fixed source. The dephasings between the antennas are used to determine the direction from which the signal is coming. This section is devoted to the derivation of the theoretical equations governing a direction finder, when all antennas are aligned. The difficulties encountered in a real world situation are emphasized.

\subsection{Theoretical approach}

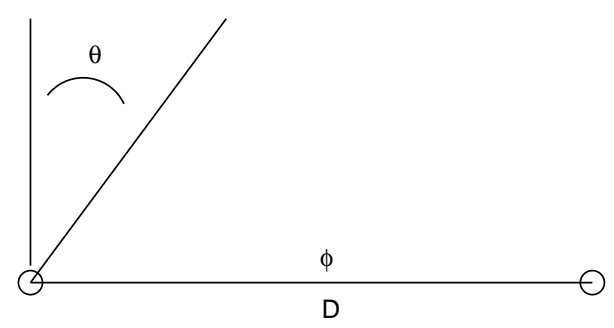

Figure 1: Two antennas direction finder

Consider a system of two antennas separated by a distance D, as represented in Figure 1. If both antennas receive an electromagnetic signal of wavelength $\lambda$ from a direction $\theta$ being in the interval ]$-\theta_{\max }, \theta_{\max }[$, the dephasing $\phi$ between the signals they respectively receive is given by

$$
\phi=\frac{2 \pi D}{\lambda} \sin \theta, \quad|\theta| \leq \theta_{\max } \leq \frac{\pi}{2}
$$

Knowing $\phi$, the determination of the angle $\theta$ is straightforward:

$$
\theta=\arcsin \left(\frac{\lambda \phi}{2 \pi D}\right)
$$

But in practice, the measured dephasing $\phi_{m}$ belongs to $[-\pi, \pi]$. The relation between $\phi_{m}$ and $\theta$ is then:

$$
\phi_{m}=\frac{2 \pi D}{\lambda} \sin \theta-2 k \pi, \text { for some } k \in \mathbb{N}
$$

The number $k$ is termed the number of turns between the antennas. This number has to be determined before the angle $\theta$ can be extracted: the information given by the two antennas is not sufficient to actually find out the direction $\theta$ from which the signal is coming.

Consider now a three antennas direction finder, as described by Figure 2.

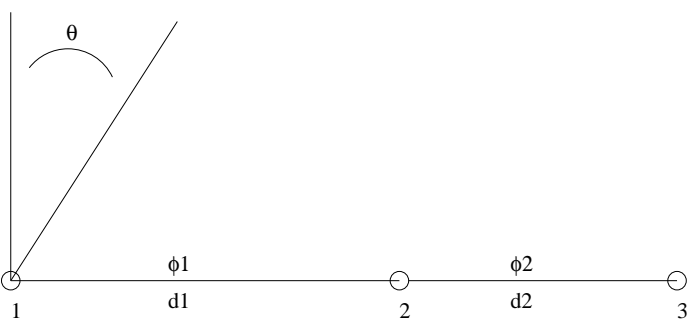

Figure 2: Three antennas direction finder

The antennas 1 and 2 (resp. 2 and 3 ) are separated by a distance $d_{1}$ (resp. $d_{2}$ ), and $\phi_{1 m}$ (resp, $\left.\phi_{2 m}\right)$ are the measured dephasing between antennas 1 and 2 (resp. 2 and 3). Applying equation (3) twice for both couples of antennas gives

$$
\phi_{2 m}=\frac{d_{2}}{d_{1}} \phi_{1 m}+2 \pi\left(\frac{d_{2}}{d_{1}} k_{1}-k_{2}\right)
$$

for some couple of integers $\left(k_{1}, k_{2}\right)$. Figure 3 shows a plot, in the $\phi_{1 m} \times \phi_{2 m}$ plane, of the solutions of (4), made of several parallel lines, one for each couple $\left(k_{1}, k_{2}\right)$.

From a couple of measures $\left(\phi_{1 m}, \phi_{2 m}\right)$ it is then possible to identify all couples $\left(k_{1}, k_{2}\right)$ :

$$
\frac{d_{2}}{d_{1}} k_{1}-k_{2}=\frac{\phi_{2 m}-\frac{d_{2}}{d_{1}} \phi_{1 m}}{2 \pi},\left|\phi_{i m}\right| \leq \pi
$$

The only one case of non-uniqueness is the case where two different couples $\left(k_{1}^{a}, k_{2}^{a}\right),\left(k_{1}^{b}, k_{2}^{b}\right)$ satisfy

$$
\frac{d_{2}}{d_{1}} k_{1}^{a}-k_{2}^{a}=\frac{d_{2}}{d_{1}} k_{1}^{b}-k_{2}^{b}, a \neq b
$$

i.e.

$$
\frac{d_{2}}{d_{1}}=\frac{k_{2}^{b}-k_{2}^{a}}{k_{1}^{b}-k_{1}^{a}}
$$




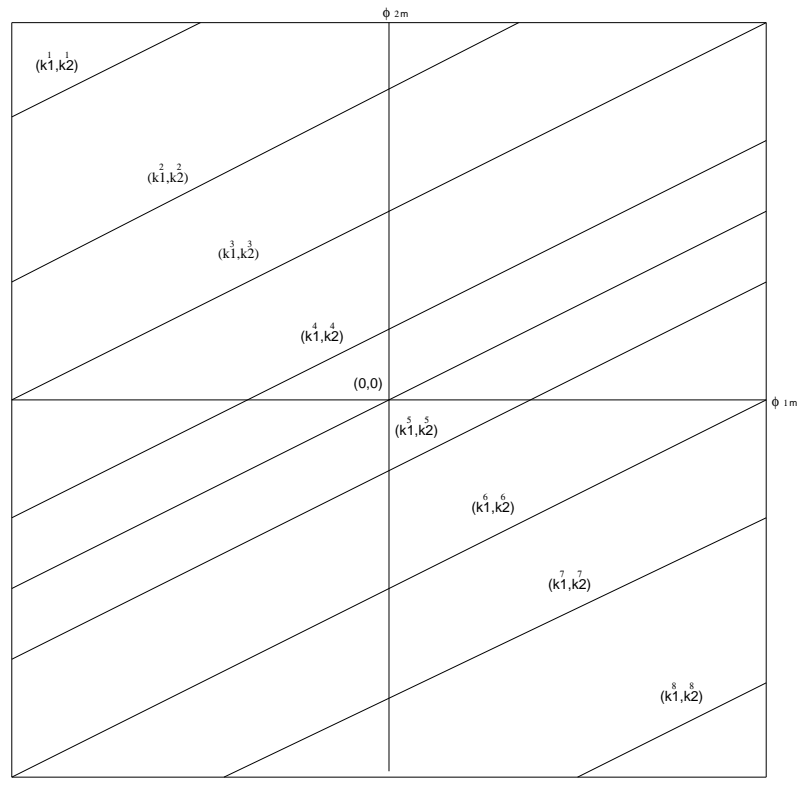

Figure 3: $\phi_{2 m}=f\left(\phi_{1 m}, k_{1}^{a}, k_{2}^{a}\right)$

So, if the distances $\left(d_{1}, d_{2}\right)$ are chosen such that $\frac{d_{2}}{d_{1}} \notin Q$, equation (5) has a unique solution $\left(k_{1}, k_{2}\right)$, which in turn allows the derivation of the angle $\theta$ from one of the equation:

$$
\theta=\arcsin \left(\frac{\lambda}{2 \pi D}\left(\phi_{1 m}+2 k_{1} \pi+\phi_{2 m}+2 k_{2} \pi\right)\right)
$$

\subsection{Real world is noisy}

But in practice, errors are made on the measures $\left(\phi_{1 m}, \phi_{2 m}\right)$. If $\delta \phi_{i}$ is the error made on $\phi_{i m}$, equation (3) becomes:

$$
\phi_{i m}=\frac{2 \pi d_{i}}{\lambda} \sin \theta-2 k_{i} \pi+\delta \phi_{i}, 1 \leq i \leq 2
$$

The relation between $\phi_{1 m}$ and $\phi_{2 m}$ is then:

$$
\phi_{2 m}=\frac{d_{2}}{d_{1}}\left(\phi_{1 m}-\delta \phi_{1}\right)+2 \pi\left(\frac{d_{2}}{d_{1}} k_{1}-k_{2}\right)+\delta \phi_{2}
$$

The measured points are generally not on one of the lines of Figure 3, but somewhere between two lines, depending on the errors. Such a situation is presented in Figure 4. The error cube corresponds to the maximum possible error of the measurement process.

When a point $\left(\phi_{1 m}, \phi_{2 m}\right)$ is measured, in order to derive $\theta$, the couple $\left(k_{1}, k_{2}\right)$ of equations (8) must be determined without ambiguity. And the simple procedure "take the nearest beam line in Figure 3" does give the correct answer if the error square of figure 4 does not intersect with the dotted line which is the median between the lines $\left(k_{1}^{a}, k_{2}^{a}\right)$ and $\left(k_{1}^{b}, k_{2}^{b}\right)^{2}$.

So, for any $\left(k_{1}^{a}, k_{2}^{a}\right),\left(k_{1}^{b}, k_{2}^{b}\right)$ both satisfying (10),

\footnotetext{
${ }^{2}$ The distance considered here is the Euclidean distance. But any distance on $\mathbb{R}^{2}$ gives the same qualitative results.
}

there exists a maximum allowable error denoted $\delta \phi_{a b}$ such that, if the maximum errors $\delta \phi_{1}$ and $\delta \phi_{2}$ on the measures of the dephasing are less than $\delta \phi_{a b}$, the error cube induced by these errors will not intersect. The value for $\delta \phi_{a b}$ is given by

$$
\delta \phi_{a b}=\pi \frac{\left|\frac{d_{2}}{d_{1}}\left(k_{1}^{b}-k_{1}^{a}\right)-\left(k_{2}^{b}-k_{2}^{a}\right)\right|}{1+\frac{d_{2}}{d_{1}}}
$$

See Annex 1 for more details.

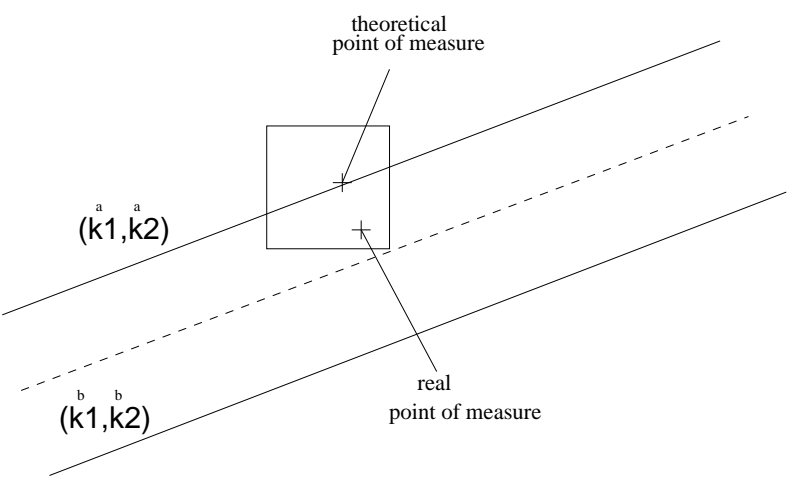

Figure 4: Error cube $\delta \phi$.

Let $\delta \phi=\min _{a \neq b} \delta \phi_{a b}$, where the minimum is taken over all $\left(k_{1}, k_{2}\right)$ satisfying relation $(9)$.

Assuming that $\delta \phi_{1}$ and $\delta \phi_{2}$ are less than $\delta \phi$, ensures that the determination of the couple $\left(k_{1}, k_{2}\right)$ of equation (8) is unambiguous (by choosing the nearest line of the theoretical beam). Thus, the determination of $\theta$ is possible, without ambiguity as well.

Remark: The distance $D$ between the two extremal antennas plays a leading role. Actually, it has an influence on the precision of the calculus of the researched angle. If $\theta_{\delta}$ is the angle calculated with errors on the measures and $\theta$ the theoretical angle:

$$
\begin{aligned}
& \theta_{\delta}=\arcsin \left(\frac{\lambda}{2 \pi}\left(\frac{\sum_{i=1}^{n-1}\left(\phi_{i m}+2 k_{i} \pi\right)}{D}+\frac{\sum_{i=1}^{n-1} \delta \phi_{i}}{D}\right)\right) \\
& \theta=\arcsin \left(\frac{\lambda}{2 \pi}\left(\frac{\sum_{i=1}^{n-1}\left(\phi_{i m}+2 k_{i} \pi\right)}{D}\right)\right) \\
& \text { and when } D \rightarrow \infty \quad \theta_{\delta} \rightarrow \theta
\end{aligned}
$$

The influence of the errors on measures becomes insignificant, that's why it is desirable to take this distance as long as possible. On the other hand, technological (and military) reasons generally impose strict restrictions on the maximum total length $D$ of the direction finder.

\subsection{The optimization problem}

Consider now the general case of $\mathrm{N}$ aligned antennas. The antennas $i$ and $i+1$ are separated by a distance $d_{i}$. The difference of phases $\phi_{i m}$ between antennas $i$ and $i+1$ are measured with some error. 
Equation (12) gives the maximum allowable error still enabling to actually solve the direction finder problem: find the direction $\theta$ of the coming signal. And this term depends on the distances $\left(d_{i}\right)$ separating the antennas. The aim of direction finder design is to find out these distances $\left(d_{i}\right)$ such that the allowable error $\delta \phi$, is maximum, thus allowing the unambiguous determination of $\theta$ even in the worst noisy possible case. The data of this optimization problem are $N$ the number of antennas ${ }^{3}, D$, the total length of the direction finder, $F$ the frequency of the signal, $\theta_{\max }$ the wanted range of visibility of incoming signals, and $d_{m i n}$, the minimum distance between antennas (imposed by the physical dimensions of the antennas).

The useful variables here are $b_{i}=\frac{d_{1}}{d_{i+1}}, i \in$ $[1, N-2]$ (with notation $b_{0}=1$ ). The optimization problem can be mathematically written as

Find $\left(b_{1}, \ldots, b_{N-2}\right), \in \mathbb{R}_{+}^{N-2}$ such that:

$$
\begin{aligned}
& \delta \phi=\inf _{\gamma \neq \alpha} \sup _{\substack{1 \leq i \leq n-2 \\
i<j \leq n-1}} \frac{\left|\frac{b i-1}{b_{j-1}}\left(k_{i}^{\alpha}-k_{i}^{\gamma}\right)-\left(k_{j}^{\alpha}-k_{j}^{\gamma}\right)\right|}{1+\frac{b_{i-1}}{b_{j-1}}} \\
& d_{i}=\frac{D}{\left(1+\sum_{j=1}^{n-2}\left(1 / b_{j}\right)\right) b_{i-1}} \geq d_{\text {min }}
\end{aligned}
$$

Some details about these formula can be found in Annex 2.

A simple plot in the case of 3 antennas for a small value of $D$ (Figure 5 ) gives an idea of the landscape of $\delta \phi$ in terms of $b=\frac{d_{1}}{d_{2}}$. Such a landscape, in this very simple case, explains why deterministic optimization algorithms fail, and why Genetic Algorithms have greater chances of success in solving this problem.

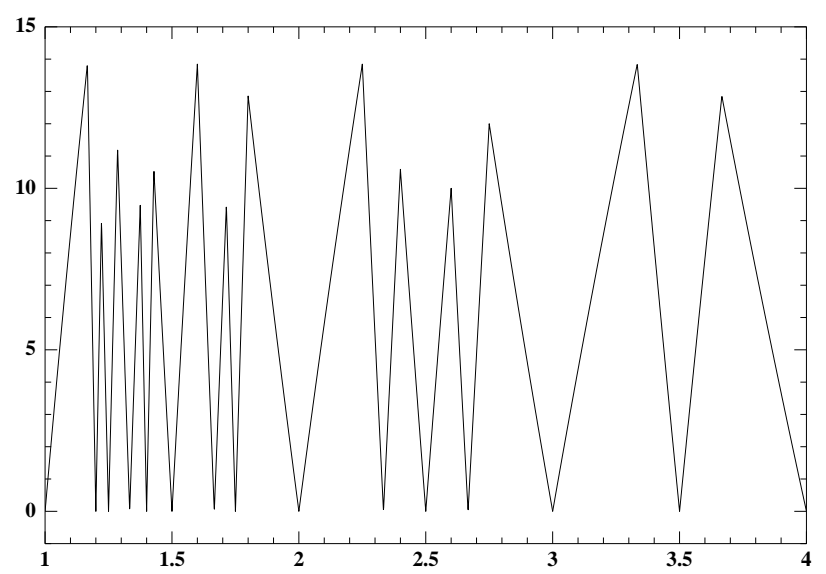

Figure 5 : Three antennas problem: Maximum allowable error vs position of the third antenna.

\footnotetext{
${ }^{3}$ which could actually be unknown, to be minimized
}

\section{The Genetic Algorithm}

The Genetic Algorithm used for the optimization problem presented in section 2.3 is a home brew GA. The general outline of the algorithm is the usual GA scheme:

- Randomly initialize the population.

- Iterate until either a prescribed number $G$ of generations is run, or $G_{\text {flat }}$ generations are run without any increase of the maximum fitness in the population:

- Select individuals according to their relative fitness.

- Recombine couples of selected individuals with probability $p_{\text {cross }}$.

- Mutate offsprings with probability $p_{m u t}$.

The different genetic operations are based on what is now becoming the standards in many real world applications of GAs dealing with real numbers:

- roulette wheel selection, with linear fitness scaling (see [2]);

- real encoding, as demonstrated powerful by [8, 7]; The genetic operators are adapted to that representation:

- The crossover operator is achieved by a linear combination of both parents (this operator is termed the intermediate recombination operator in Evolution Strategies, see [9, 1]).

The offspring of the two parents $\left(x_{1}, \ldots, x_{N}\right)$ and $\left(y_{1}, \ldots, y_{N}\right)$ is $\left(\alpha x_{1}+(1-\alpha) y_{1}, \ldots, \alpha x_{N}+\right.$ $\left.(1-\alpha) y_{N}\right)$ for some $\alpha$ randomly chosen uniformly in $[-0.5,1.5]$.

- The mutation operator is performed by adding some Gaussian noise to the some randomly chosen coordinate(s) of the chromosome. The main differences with the mutation scheme of Evolution Strategies [9, 1] is that each coordinate has a probability of being changed, and the evolution scheme for the standard deviations of the Gaussian noise is a geometric decrease instead of an adaptative evolution.

- When some niching scheme is needed, the sharing scheme as described in [3] is used.

- For all runs, the stopping criterion is either a maximum number of generations, or a prescribed number of generations without any improvement, whichever comes first.

The actual parameters used are: $p_{\text {cross }}=0.65, p_{\text {mut }}=0.075$ 


\section{Numerical results}

This section is devoted to the numerical results obtained using the GA briefly presented in section 3 on the direction finder optimization problem of section 2.3 .

\begin{tabular}{|c|c|c|c|c|c|}
\hline $\mathrm{N}^{\mathrm{D}}$ & 100 & 200 & 400 & 800 & 1600 \\
\hline \multirow[t]{2}{*}{4} & $\begin{array}{l}\mathrm{b} 1=1.2 \\
\mathrm{~b} 2=3\end{array}$ & $\begin{array}{l}\mathrm{b} 1=3.2 \\
\mathrm{~b} 2=4\end{array}$ & $\begin{array}{l}\mathrm{b} 1=5.143 \\
\mathrm{~b} 2=6\end{array}$ & $\begin{array}{l}\mathrm{b} 1=9 \\
\mathrm{~b} 2=10.125\end{array}$ & $\begin{array}{l}\mathrm{b} 1=13 \\
\mathrm{~b} 2=14.083\end{array}$ \\
\hline & $\delta \phi=45$ & $\delta \phi=34.285$ & $\delta \phi=25.11$ & $\delta \phi=18$ & $\delta \phi=12.85$ \\
\hline 5 & $\begin{array}{l}\mathrm{b} 1=2.052 \\
\mathrm{~b} 2=2.563 \\
\mathrm{~b} 3=3.376\end{array}$ & $\begin{array}{l}\mathrm{b} 1=2.494 \\
\mathrm{~b} 2=4.734 \\
\mathrm{~b} 3=7.627\end{array}$ & $\begin{array}{l}\mathrm{b} 1=2.382 \\
\mathrm{~b} 2=3.866 \\
\mathrm{~b} 3=4.439\end{array}$ & $\begin{array}{l}\mathrm{b} 1=2.069 \\
\mathrm{~b} 2=4.008 \\
\mathrm{~b} 3=5.48\end{array}$ & $\begin{array}{l}\mathrm{b} 1=3.051 \\
\mathrm{~b} 2=5.669 \\
\mathrm{~b} 3=7.422\end{array}$ \\
\hline \multirow[t]{2}{*}{6} & $\begin{array}{l}\delta \phi=55.84 \\
\mathrm{~b} 1=1.185 \\
\mathrm{~b} 2=1.434 \\
\mathrm{~b} 3=1.946 \\
\mathrm{~b} 4=2.104\end{array}$ & $\begin{array}{l}\delta \phi=49.42 \\
\mathrm{~b} 1=1.398 \\
\mathrm{~b} 2=2.736 \\
\mathrm{~b} 3=3.785 \\
\mathrm{~b} 4=5.472\end{array}$ & $\begin{array}{l}\delta \phi=40.66 \\
\mathrm{~b} 1=2.756 \\
\mathrm{~b} 2=4.707 \\
\mathrm{~b} 3=5.324 \\
\mathrm{~b} 4=8.177\end{array}$ & $\begin{array}{l}\delta \phi=32.36 \\
\mathrm{~b} 1=3 \\
\mathrm{~b} 2=4.546 \\
\mathrm{~b} 3=5.4 \\
\mathrm{~b} 4=7.659\end{array}$ & $\begin{array}{l}\delta \phi=26.21 \\
\mathrm{~b} 1=2.255 \\
\mathrm{~b} 2=3.03 \\
\mathrm{~b} 3=4.017 \\
\mathrm{~b} 4=5.384\end{array}$ \\
\hline & $\delta \phi=64.02$ & $\delta \phi=60$ & $\delta \phi=47.91$ & $\delta \phi=44.96$ & $\delta \phi=37.79$ \\
\hline
\end{tabular}

Figure 6 : Optimal placements for antennas found by GAs. $N$ is the number of antennas, and $D$ the total length of the apparatus.

Fortunately, these results confirm some points that direction finder experts have known for long: when $D$ increases, the maximum allowable error $\delta \phi$ decreases, and the only way to increase $\delta \phi$ is to use more antennas. The only difference is that now, the optima with up to 10 antennas can be computed. Moreover, the results of Figure 6 also demonstrate that the incremental method is definitely useless when the number of antennas increases, as the optimum solution for $N+1$ antennas is not a simple addition of one antenna to any optimum for $N$ antennas.

But even more insight in the problem of direction finder can be gained from a careful look at the numerical results obtained by the GAs, as is demonstrated in the next section.

\section{New theoretical insight}

One of the main advantages of GAs, apart from being able to solve difficult optimization problems untractable with standard optimization methods, is the ability to find out multiple quasi-optimal solutions to a given problem. This can be achieved either incidently, by running the same algorithm with different random initializations of the population, of more systematically by adding some niching technique to the basic GA. The sharing scheme (see [3]) is used in the GA presented in section 3 .

From the equations giving $\delta \phi$ (see section 2.3 and Annex 2), it seems clear that some kind of regularity exists in the problem. However, no direction finder expert has been able to precisely identify any theoretical relations between different optima.

But a close look at many results obtained for

\begin{tabular}{|c|c|c|c|c|c|}
\hline 12.5 & 25 & 50 & 100 & 200 & 400 \\
\hline $\mathrm{b}=1.5$ & $b=3$ & $\begin{array}{l}b=1.333 \\
b=2.5\end{array}$ & $\begin{array}{l}b=1.1667 \\
b=2.4999 \\
b=12\end{array}$ & $\begin{array}{l}b=2.125 \\
b=3.1666 \\
b=5.25\end{array}$ & $\begin{array}{l}b=1.0417 \\
b=3.083 \\
b=5.125 \\
b=7.1666 \\
b=11.25\end{array}$ \\
\hline$\delta \phi=72$ & $\delta \phi=45$ & $\delta \phi=25.71$ & $\delta \phi=13.84$ & $\delta \phi=7.2$ & $\delta \phi=3.67$ \\
\hline
\end{tabular}

Figure $7: G A$ results for $D=2^{\alpha} 12.5, N=3$

the 3 antennas problem, where $\delta \phi$ is a function of 1 variable $\left(\frac{d_{1}}{d_{2}}\right)$ gave hints that finally lead to an answer. The following relations give all the maxima in term of the data:

$$
\begin{aligned}
& \text { Let } \frac{D}{\lambda} \sin \theta_{\text {max }} \in \mathbb{N}: \\
& \begin{aligned}
\forall k_{1 \text { max }} \in \mathbb{N}, 0 \leq k_{1 \text { max }}<\frac{D}{\lambda} \sin \theta_{\text {max }} \\
b=\frac{2 k_{1 \max }+1}{2\left(\frac{D}{\lambda} \sin \theta_{\max }-k_{1 \max }\right)} \\
\delta \phi=\frac{\pi}{1+2 \frac{D}{\lambda} \sin \theta_{\max }}
\end{aligned}
\end{aligned}
$$

The number of maxima $\mathrm{M}$ is:

$$
M\left(D, \lambda, \theta_{\max }\right)=2 \frac{D}{\lambda} \sin \theta_{\max }
$$

The technical details can be found in Annex 3 .

Remark: The component of the maxima for $\frac{D}{\lambda} \sin \theta_{\max } \in \mathbb{R}$ can be found at the end of the Annex3.

Let's apply the relations $(14),(15),(16)$ for the case $\mathrm{D}=100 \mathrm{~mm}, \mathrm{~F}=18 \mathrm{Ghz}(\lambda=16.667 \mathrm{~mm})$, $\theta_{\max }=\frac{\pi}{2}, d_{\min }=0$

$\frac{D}{\lambda} \sin \theta_{\max }=6$

$\delta \phi$ can be immediately derivated :

$\delta \phi=\frac{\pi}{13} \approx 13.846154$

this maximal value is obtained for the following $b^{i}$, extracted for $0 \leq k 1 \max \leq 5$

$k_{1 \text { max }}=0 \Rightarrow b^{0}=\frac{1}{12} \approx 0.0833333$

$k_{1 \max }=1 \Rightarrow b^{1}=\frac{3}{10}=0.3$

$k_{1 \text { max }}=2 \Rightarrow b^{2}=\frac{5}{8}=0.625$

$k_{1 \text { max }}=3 \Rightarrow b^{3}=\frac{7}{6} \approx 1.1666667$

$k_{1 \max }=4 \Rightarrow b^{4}=\frac{9}{4}=2.25$

$k_{1 \max }=5 \Rightarrow b^{5}=\frac{11}{2}=5.5$

Together with their inverse values (if $\mathrm{b}$ is a solution, obviously $\frac{1}{b}$ is too)

$M\left(100,16.6667, \frac{\pi}{2}\right)=12$.

We can also notice that maxima for a given distance $D=2^{\alpha} 12.5$ can also be found by addicting an integer, or dividing the decimal part by 2 of 
the maxima $D=2^{\alpha-1} 12.5$ (figure 6 ). This is in fact the observation of these links which lead us to study a theoretical approach, and make us found out the expression of $\mathrm{b}$ and $\delta \phi$ in function of the given informations.

The derivation of the same kind of results for any number of antennas is part of ongoing work, and the technical difficulties are almost overwhelmed (see [15]).

\section{Conclusion and future work}

An application of GA optimization to direction finder optimization has been presented. The state of the art of electromagnetism engineering was limited by the use of classical optimization methods to 3 or 4 aligned antennas. The evolutionary optimization is able to handle the case of up to 10 aligned antennas. Moreover, the ability of GAs to find multiple optima lead to the derivation of a theoretical analysis of the problem, through a careful look at all optima. Though this theoretical analysis has no $a$ posteriori relation with GAs, it had not been found out before numerical optimization gave hints that finally lead to the solution.

Regarding the numerical optimization of direction finders, ongoing work is concerned with more general configurations of the antennas.

The first results on coplanar antennas is a strict extension of the aligned case, though the technical details of the derivation of the fitness function are awkward looking (see [15] for all the details). The first results obtained with the same method are presented in Figure 8, in the case where the antennas must lie on a given half-circle. The more general case of 3-dimensional direction finders, where both the bearing angle and the site angle are to be determined by the direction finder is currently under investigation.

Moreover, the same kind of theoretical result then the one presented in section (5) has been obtained for the general case of $\mathrm{N}$ aligned antennas. But the extension of these results to more general situations seems more difficult.

Finally, electromagnetism engineering offers many optimization problems whose characteristics appeal for evolutionary computation, e.g. diagram synthesis, partitioning of networks of antennas.

\section{References}

[1] Bäck T. and Schwefel H.-P., An Overview of Evolutionary Algorithms for Parameter Optimization, Technical Report, University of Dort-

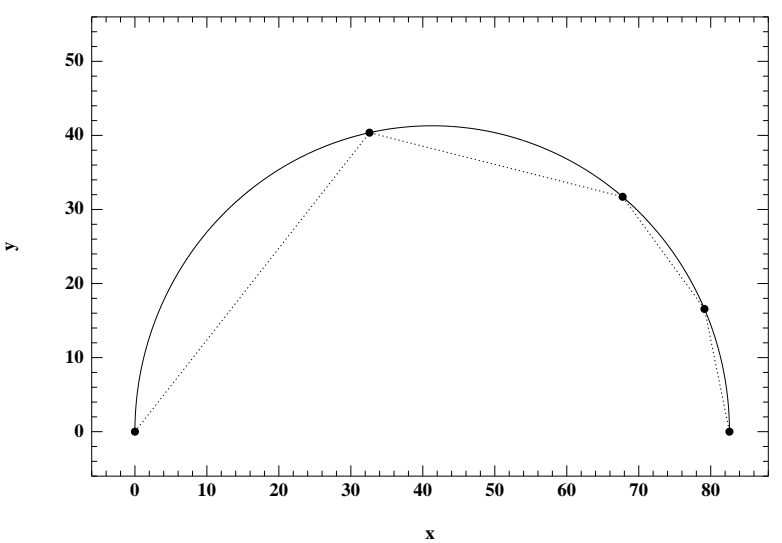

Figure 8: Five antennas direction finder on a semicircular form

mund, 1994.

[2] Golberg D.E., Genetic algorithms in search, optimization and machine learning. Addisson Wesley, 1989.

[3] D. E. Goldberg, J. Richardson, Genetic algorithms with sharing for multi-modal function optimization. in J.J. Grefenstette Editor, Proceedings of the $2^{\text {nd }}$ International Conference on Genetic Algorithms, Lawrence Erlbaum Associates, pp 41-49, 1987.

[4] J. Holland, Adaptation in natural and artificial systems, University of Michigan Press, Ann Harbor, 1975.

[5] K. A. De Jong, Are genetic algorithms function optimizers ? in Proceeding of the second conference on Parallel Problem Solving from $\mathrm{Na}$ ture, Free University of Brussel, North Holland Publishers, pp 3-13, 1992

[6] Mitchell M. and Holland J.H., When will a genetic algorithm outperform hill-climbing ? In Proceedings of the $3^{\text {rd }}$ International Conference on Genetic Algorithms, George Mason University, Morgan Kauffman Publishers, June 4-7 1989.

[7] Michalewicz Z., Genetic Algoritms+Data Structures=Evolution Programs. Springer Verlag. 1992.

[8] Radcliffe N. J., Equivalence class analysis of genetic algorithms. Complex Systems, 1991.

[9] H.-P. Schwefel, Numerical Optimization of Computer Models. John Wiley \& Sons, NewYork, 1981.

[10] Jacobs, E., and Ralston, E.W.(1981) Ambiguity resolution in interferometry. IEEE Transactions on Aerospace and Electronic Systems, AES-17 (Nov. 1981), 766-780. 
[11] Dybdal R.B., Monopulse Resolution of Interferometric Ambiguities. IEEE Transactions on Aerospace and Electronic Systems AES-22 (2), March 1986.

[12] Guy J.R.F and Davies D.E.N., Studies of the Adcock direction finder in terms oh phasemode excitations around circular array. The radio and Electronic Engineer, January 1983.

[13] Kummer W.H., Broad-Band Microwave Electronically Scanned Direction Finder IEEE Transactions on Antennas Propagation, AP31, January 1983.

[14] Custin A.R., Performance of an interferometer angle measuring receiver against non lineof-sight transmitters IEE Proceedings, 130 (7), Part F, December 1983.

[15] Taïeb L., Optimisation en électromagnétisme par algorithmes génétiques. Thèse de l'Ecole Polytechnique, spécialité Analyse Numérique. In preparation.

Annex $1: \delta \phi_{a b}=\pi \frac{\left|\frac{d_{2}}{d_{1}}\left(k_{1}^{a}-k_{1}^{b}\right)-\left(k_{2}^{a}-k_{2}^{b}\right)\right|}{1+\frac{d_{2}}{d_{1}}}$

$$
\begin{aligned}
& 2 \pi\left(\frac{d_{2}}{d_{1}} k_{1}^{a}-k_{2}^{a}\right)=\phi_{2 m}-\delta \phi_{2}^{a}-\frac{d_{2}}{d_{1}}\left(\phi_{1 m}-\delta \phi_{1}^{a}\right) \\
& 2 \pi\left(\frac{d_{2}}{d_{1}} k_{1}^{b}-k_{2}^{b}\right)=\phi_{2 m}-\delta \phi_{2}^{b}-\frac{d_{2}}{d_{1}}\left(\phi_{1 m}-\delta \phi_{1}^{b}\right)
\end{aligned}
$$

From these two relations it is possible in eliminating the measures to express the differences of turns $\triangle k_{p}^{a b}=k_{p}^{b}-k_{p}^{a}$ in function of the difference of the errors committed $\triangle \phi_{p}^{a b}=\delta \phi_{p}^{b}-\delta \phi_{p}^{a}$ :

$$
2 \pi\left(\frac{d_{2}}{d_{1}} \triangle k_{1}^{a b}-\triangle k_{2}^{a b}\right)=\frac{d_{2}}{d_{1}} \triangle \delta \phi_{1}^{a b}-\triangle \delta \phi_{2}^{a b}
$$

Between two couples $\left(k_{1}^{a}, k_{2}^{a}\right),\left(k_{1}^{b}, k_{2}^{b}\right)$ there is no ambiguity if for a $\delta \phi^{a b} \in \mathbb{R}$ such as:

$\forall \quad\left|\delta \phi_{p}^{a}\right|,\left|\delta \phi_{p}^{b}\right|<\delta \phi^{a b}, \quad 1 \leq p \leq 2$

$$
2 \pi\left(\frac{d_{2}}{d_{1}} \triangle k_{1}^{a b}-\triangle k_{2}^{a b}\right) \neq \frac{d_{2}}{d_{1}} \triangle \delta \phi_{1}^{a b}-\triangle \delta \phi_{2}^{a b}
$$

which implies:

$$
\pi\left|\frac{d_{2}}{d_{1}} \triangle k_{1}^{a b}-\triangle k_{2}^{a b}\right| \geq\left(1+\frac{d_{2}}{d_{1}}\right) \delta \phi^{a b}
$$

and then:

$$
\delta \phi^{a b}=\pi \frac{\left|\frac{d_{2}}{d_{1}} \triangle k_{1}^{a b}-\triangle k_{2}^{a b}\right|}{1+\frac{d_{2}}{d_{1}}}
$$

\section{Annex 2 :}

The basic relations relations of the problem are:

$b_{i}=\frac{d_{1}}{d_{i+1}}, b_{0}=1$ $\phi_{i m}=\frac{2 \pi d_{i}}{\lambda} \sin \theta-2 k_{i}^{\alpha} \pi+\delta \phi_{i}$

$1 \leq i \leq n-1, k_{i}^{\alpha} \in N,\left|\delta \phi_{i}\right| \leq \delta \phi$

$\phi_{j m}=B(i, j)\left(\phi_{i m}+2 \pi k_{i}^{\alpha}-\delta \phi_{i}\right)-2 \pi k_{j}^{\alpha}+\delta \phi_{j}$

$B(i, j)=\frac{b_{i-1}}{b_{j-1}}$

$1 \leq i \leq n-2, i<j \leq n-1$

$-\pi \leq \phi_{i m} \leq \pi$

The preceeding relations imply that for each measured dephasing $\phi_{i m}$, there is a finite number of turns which allow to find out the theoretical dephasing $\phi_{i}$ :

$\left|k_{i}\right| \leq E\left[\frac{d_{i}}{\lambda} \sin \theta_{\max }+\frac{1}{2}\left(1+\frac{\delta \phi}{\pi}\right)\right], k_{i} \in N$ with:

$d_{i}=\frac{D}{\left(1+\sum_{j=1}^{n-2}\left(1 / b_{j}\right)\right) b_{i-1}}$

$\lambda=\frac{c}{F}$

The previous relations show that among all the possibilities of turns, we only consider those which respect the condition :

$$
\sup _{\substack{1 \leq i \leq n-2 \\ i<j \leq n-1}} \frac{\left|\frac{b_{i-1}}{b_{j-1}} k_{i}^{\alpha}-k_{j}^{\alpha}\right|}{1+\frac{b_{i-1}}{b_{j-1}}} \leq \frac{1}{2}\left(1+\frac{\delta \phi}{\pi}\right)
$$

Generalizing what have been done for 4 antennas in the Annex1, gives equations (12) and (13).

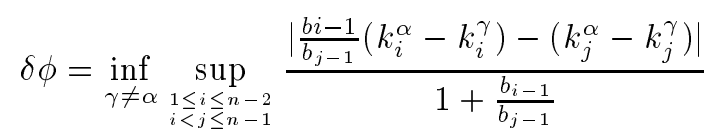

$$
\begin{aligned}
& d_{i}=\frac{D}{\left(1+\sum_{j=1}^{n-2}\left(1 / b_{j}\right)\right) b_{i-1}} \geq d_{m i n}
\end{aligned}
$$

Annex 3 : Analytic relations for 3 antennas.

The problem studied is the one modelized in Annex2 for $\mathrm{N}=3$ with last condition replaced by

$$
\frac{\left|\frac{1}{b} k_{1}^{\alpha}-k_{2}^{\alpha}\right|}{1+\frac{1}{b}}<\infty
$$

all possible combinations of $\left(k_{1}, k_{2}\right)$ satisfying $\left|k_{i}\right| \leq$ $k_{\text {imax }}$ are considered (this does not change any result).

The aim is to find out the couple $(b, \delta \phi)$ such that $\delta \phi$ is maximal. The data are the distance $D$ between the two extremal antennas, the wavelength $\lambda$, the maximal angle for incoming signals $\theta_{\max }$, and the minimal distance between two antennas set here to $0\left(d_{\min }=0\right)$.

If $k_{1 \max }$ and $k_{2 \max }$ are the maximum numbers of turns that can generate ambiguities, the 
maximum allowable error is given by:

$$
\delta \phi=\inf _{\substack{\left|\triangle k_{1}\right| \leq 2 k_{1 \max } \\\left|\triangle k_{2}\right| \leq 2 k_{2 \max }}} \pi \frac{\left|\triangle k_{1}-b \triangle k_{2}\right|}{1+b}
$$

Case 1: $k_{2 \max }=0$ with $\frac{D}{\lambda} \sin \theta_{\max } \in \mathbb{R}$

$$
\delta \phi=\inf _{0<\left|\triangle k_{1}\right| \leq 2 k_{1 \max }} \pi \frac{\left|\triangle k_{1}\right|}{b+1}
$$

$\delta \phi$ is minimal when $\left|\triangle k_{1}\right|=1$

then the value to maximise is $\delta \phi=\frac{1}{1+b}$

$\mathrm{b}$ has to be minimise but respecting the condition $k_{2 \max }=0$, that implies:

$\frac{D}{\lambda(b+1)} \sin \theta_{\max }+\frac{1}{2}\left(1+\frac{\delta \phi}{\pi}\right)<1$

reporting $\delta \phi=\frac{1}{b+1}$ in this relation, it comes:

$b>2 \frac{D}{\lambda} \sin \theta_{\max }$

Finaly:

$$
b \rightarrow 2 \frac{D}{\lambda} \sin \theta_{\max } \quad \delta \phi \rightarrow \frac{\pi}{1+2 \frac{D}{\lambda} \sin \theta_{\max }}
$$

Case 2: general case with $\frac{D}{\lambda} \sin \theta_{\max } \in \mathbb{N}$

Lets find out $b$ such as $b=a_{1} R_{b}$, $a_{1} \in N, R_{b} \in Q$ and verifying for $a_{2} \in N^{+}$

$$
\begin{aligned}
& \left(2 k_{2 \max }-a_{2}\right) R_{b}=1-R_{b} \\
& \left(2 k_{2 \max }-a_{2}+1\right) a_{1} R_{b} \geq 2 k_{1 \max }+R_{b}
\end{aligned}
$$

it comes:

$$
\begin{gathered}
R_{b}=\frac{1}{2 k_{2 \max }+1-a_{2}} \\
\delta \phi=\frac{\pi R_{b}}{a_{1} R_{b}+1}=\frac{\pi}{2 k_{2 \max }+1+a_{1}-a_{2}}
\end{gathered}
$$

Using (3.2):

$$
a_{1} \geq 2 k_{1 \max }+\frac{1}{2 k_{2 \max }+1-a_{2}}
$$

As $a_{1} \in N$

$$
a_{1}=2 k_{1 \max }+1
$$

Therefore:

$$
\begin{gathered}
b=\frac{2 k_{1 \max }+1}{2 k_{2 \max +1-a_{2}}} \\
\delta \phi=\frac{\pi}{2\left(k_{1 \max }+k_{2 \max }+1\right)-a_{2}}
\end{gathered}
$$

To maximize $\delta \phi, a_{2}$ has to be maximize but respecting $k_{1 \max }$ and $k_{2 \max }$ used before. when $a_{2}$ increases, $b$ increases too, and then $k_{1 \max }, a_{2}$ has to verify:

$$
\frac{b D \sin \theta_{\max }}{\lambda(b+1)}+\frac{1}{2}\left(1+\frac{\delta \phi}{\pi}\right)<k_{1 \max }+1
$$

and then:

$a_{2} \leq 2\left(k_{1 \max }+k_{2 \max }+1\right)-\frac{1}{2 k_{1 \max }}-2 \frac{D}{\lambda} \sin \theta_{\max }$

as $a_{2} \in \mathbb{N}, \frac{D}{\lambda} \sin \theta_{\max } \in \mathbb{N}$

$$
a_{2}=2\left(k_{1 \max }+k_{2 \max }\right)+1-2 \frac{D}{\lambda} \sin \theta_{\max }
$$

finaly:

$$
b=\frac{2 k_{1 \max }+1}{2\left(\frac{D}{\lambda} \sin \theta_{\max }-k_{1 \max }\right)} \quad \delta \phi=\frac{\pi}{1+2 \frac{D}{\lambda} \sin \theta_{\max }}
$$

With $k_{1 \max }=0$, we find back the inverse value of the Case 1 of this Annex. If $b$ is an optimum $\frac{1}{b}=\frac{d_{2}}{d_{1}}$ is an optimum too. Then the total number of maxima $M$ is:

$$
M\left(D, \lambda, \theta_{\max }\right)=2 \frac{D}{\lambda} \sin \theta_{\max }
$$

The component of the maxima for $\frac{D^{i}}{\lambda} \sin \theta_{\max } \in \mathbb{R}$ seams to respect the following rules:

Let D such as $\frac{D}{\lambda} \sin \theta_{\max } \in \mathbb{N}$

$\exists D^{1}>D, \frac{D^{1}}{\lambda} \sin \theta_{\max } \in \mathbb{R}$

such as from $D+\epsilon$ to $D^{1}$

$b_{D^{1}}^{i}=b_{D}^{i}$, for all $i, i \neq 0, i \neq \frac{D}{\lambda} \sin \theta_{\max }$

$\delta \phi=\frac{\pi}{1+2 \frac{D}{\lambda} \sin \theta_{\max }}$

$M=2\left(\frac{D}{\lambda} \sin \theta_{\max }-1\right)$

The two values of $b$ given by the Case 1 of this Annex are lost.

$\exists D^{2}>D^{1}, \frac{D^{2}}{\lambda} \sin \theta_{\text {max }} \in \mathbb{R}$

such as from $D^{1}+\epsilon$ to $D^{2}$

$b_{D^{2}}^{i}=b_{D}^{i}$, for some $\mathrm{i}$

$\delta \phi=\frac{\pi}{1+2 \frac{D}{\lambda} \sin \theta_{\max }}$

$M<2\left(\frac{D}{\lambda} \sin \theta_{\max }-1\right)$

$\exists D^{3}>D^{2} \frac{D^{3}}{\lambda} \sin \theta_{\text {max }} \in \mathbb{R}$

such as from $D^{2}+\epsilon$ to $D^{3}$

$b \in \mathbb{R}, M=1$

In this case the previous relations that give $(b, \delta \phi)$ can not be used because $b$ has been supposed belong to $\mathrm{Q}$. 